\title{
Suaves asimetrías
}

Este articulo fue publicado originalmente por la revista Casabella de abril de 2001 ( $\left.n^{\circ} 688\right)$, a propósito de una crítica a las obras finalistas para la $2^{a}$ versión del premio Mies Van der Rohe de Arquitectura Latinoamericana, otorgado por la Fundació Mies Van der Rohe de Barcelona. Nos parece importante conservar como documento -y en castellano- la visión sobre el concurso, que reflexiona sobre cierta actitud del bemisferio norte respecto a las ex-colonias del sur: La iniciativa del premio Latinoamericano fue suspendida indefinidamente por sus organizadores en el año 2002.

This article was first published on Casabella, on April 2001 $\left(n^{\circ} 688\right)$, as a critic vision about the 2 nd version of the Mies Van der Robe award for Latin-American Architecture, granted by the Fundació Mies Van der Rohe of Barcelona. We think that is important to conserve this vision about the prize, which reflects on certain attitude of the North hemisphere towards to the ex-colonies of the south. The initiative of the Latin American award was suspended indefinitely by its organizers in 2002.

"Para estos establecimientos no convienen bixos de la tierra por que es muy difícil sacarlos de aquell costumbre tan radicada aun en contravención de las más sagradas disposiciones; falta en ellos aquel modo de pensar tan puro, sincero, e imparcial que ay en España, y aun estos mismos connaturalizados de algún tiempo en estos payses llegan a bavituarse en iguales o peores costumbres"

Carta de Francisco de Viedma, Intendente de Cochabamba, a J osŽ de Głlvez, Ministro de Indias de
Carlos III: noviembre de 1784.

Las cuestiones que me han hecho declinar sendas invitaciones a participar del Premio Latinoamericano Mies van der Rohe como experto y como jurado son otras, y he accedido a escribir estas líneas porque estas consecuencias no son tan inofensivas. Creo que más allá de la buena voluntad de sus promotores y en la medida en que no se modifiquen sus procesos y criterios, la versión para Latinoamérica del Premio otorgado por la Fundación Mies van der Rohe, contiene por su propia dinámica, de manera estructural y no aleatoria, una minusvalorización del campo cultural de la comunidad a la que aparentemente se trata de favorecer. En otras palabras, me parece que la versión para Latinoamérica del premio tiene una tonalidad paternalista cuya necesidad y su misma existencia me resigno a aceptar.

(Una comparación puede ayudarnos a comprender la disparidad política -que de eso se trata- a la que estoy haciendo referencia. Basta imaginar la excitación con que la cultura italiana, francesa o alemana esperaría los premios a la mejor obra de arquitectura europea otorgados cada dos años por una hipotética "Fundación Charles Edouard Jeanneret" con base en la casa construida por Le Corbusier para el Dr. Currutchet en la capital de la Provincia de Buenos Aires, con el auspicio del Mercosur). He agrupado mis observaciones en una lista de cuatro suaves asimetrías entre las dos versiones del premio que quisiera poner a consideración del lector. Llamo "asimetrías fuertes" a las que caracterizaban a la administración de los asuntos americanos durante los largos siglos de la dominación colonial. Sé que la referencia puede resultar antipática, exagerada, e impropia de los tiempos que corren, pero la menciono porque no logro descartarla de mi imaginario. Después de todo, el neologismo "sudaca" para designar a los latinoamericanos no es un invento mío sino la despectiva manera con la que en la sociedad española contemporánea se nos designa ("sudacas", para el lector no hispanoparlante, son esos cargosos primos pobres que, en otra época corridos por sus dictadores, se han venido a Europa a disputarse las migajas de la actual prosperidad post-industrial). Aludiendo a las "asimetrías fuertes", los textos de historia recuerdan que a fines del siglo XVIII en la administración de los asuntos del Río de la Plata había un $64 \%$ de peninsulares y apenas un $29 \%$ de porteños. Afortunadamente han ocurrido muchas cosas buenas desde entonces entre ambas partes, y las asimetrías fuertes han quedado atrás para siempre. Describiré las suaves.

Uno. Observando la composición del jurado del premio quizás se nos podría decir que las cosas no son tan tremendas. Después de todo, de los nueve que lo otorgaron, los latinoamericanos son casi la mitad -cuatro- mientras que solamente cinco, los restantes, provienen de distintos países europeos y de Estados Unidos. Es lo que llamo una asimetría suave. Que ciertamente adquiere mayor vigor cuando se observa que a su vez ninguno de los nueve miembros del jurado que otorga el premio europeo proviene de América Latina.

Me pregunto cuál es la base de esta diferencia y no logro organizar alguna respuesta razonable y/o politically correct. Es cierto que se trata de un premio otorgado por una institución europea, pero entonces: ¿Qué hacen en el jurado latinoamericano dos miembros provenientes de Estados Unidos? ¿No bastaría con un representante de la institución promotora -el secretario, por caso- para cumplir un comprensible requisito formal?

Visto en el otro sentido: ¿Por qué motivo no hay ni hubo hasta ahora ningún miembro latinoamericano en el jurado europeo? No ciertamente por razones de estricta pertinencia geográfica. En ese caso: ¿Qué hacía en uno de ellos un prestigioso colega japonés?

Dos. La segunda asimetría atraviesa los enunciados con que la Fundación Mies van der Rohe presenta ambos premios. Uno de ellos "estudia, documenta y reconoce la excelencia de la arquitectura" en su respectivo territorio; con el otro en cambio se busca "fomentar el conocimiento y estimular los intercambios arquitectónicos y culturales" entre su territorio y otro continente. Tampoco aquí logro imaginar respuestas consistentes para la leve diferencia: ¿por qué en el segundo caso no se menciona la "excelencia", y basta en cambio con conocer y estimular intercambios?, ¿por qué en el primer caso no interesa tanto recibir esos estímulos externos?, ¿qué clase de intercambios se propone si no se los busca en ambas partes presuntamente interesadas? No sé si es necesario aclararlo, pero el premio latinoamericano no es el primero sino el segundo de los aludidos.

Tres. Como lo indica su nombre, el Premio Europa toma en cuenta una unidad geográfico política. El Premio Latinoamérica, en cambio, toma en cuenta una unidad cultural, no geográfica: parece bizarro incluir en América Latina al Canadá francés o a Surinam; y como se sabe, desde un punto de vista geográfico América se divide en norte (que incluye a México), central y caribe, y sur. De no existir esta suave asimetría quizás lo razonable sería proponer: o bien un Premio América o, viceversa, distintos premios regional-culturales: Premio Europa Mediterránea o Nórdica. O, más parecido al del otro lado del Atlántico, Premio Europa Latina.

Encuentro un par de respuestas posibles para este sutil desequilibrio. Ninguna me convence.

Primera: el origen español de la iniciativa, esto es: la natural relación de España con sus ex-colonias del ultramar occidental. Pero esa naturalidad, para algunos comprensible en el ámbito de la lengua, no lo es tanto en el de la arquitectura: si algo caracteriza a los procesos de modernización de esta disciplina en los países al sur de Estados Unidos es precisamente su escasa o inexistente relación con la historia de España.

Segunda: No podría otorgarse un Premio América simplemente porque la producción entre el Norte y el Sur del continente no puede compararse: perdería siempre el Sur. En esta hipótesis el Premio Latinoamérica sería un acto de "discriminación positiva”, una suerte de premio especial (no equiparable con la "excelencia" a secas del premio europeo), inspirado en una loable vocación de dar, también, un espacio a los más débiles.

Creo que se trata de un razonamiento falso. Aceptable si se buscara valorar la cantidad de producción arquitectónica en su conjunto (obviamente mayor en el norte que en el sur). Aceptable también si se tratara de comparar trayectorias individuales porque la histórica inestabilidad del Sur las dificulta o las trunca, muchas veces con violencia. Pero en nuestro caso se busca señalar, cada dos años, una, y sólo una, obra de gran calidad.

Y me pregunto: ¿Ganaría siempre una obra del 
norte si hiciéramos el ejercicio de dar hipotéticos premios a lo largo del siglo XX? En 1936, por caso: ¿Se produjo en el norte una obra más valiosa que el Ministerio de Educación de Río de Janeiro?; o en 1948 ¿Hubo en el norte una pieza comparable a la casa del puente de Amancio Williams en Mar del Plata?; o en 1952: ¿Alguien construyó en América una obra mejor que la plaza cubierta y el auditorio de la ciudad Universitaria de Caracas?; y ¿Cuál es la obra del norte que en 1970 le hubiera quitado a Rogelio Salmona el Premio América por sus Torres del Parque en Bogotá?

No importa cuáles sean nuestras respuestas a estas preguntas, pero es evidente que no nos resultaría sencillo decidirlas. Es que a la hora de emitir un juicio sobre la calidad de una pieza de arquitectura, los sistemas políticos, las condiciones económicas, o la biografía de su autor importan bien poco. Afortunadamente o no, el poder y el dinero no tienen efecto en su densidad ni en su capacidad de iluminarnos.

Cuatro. El proceso de selección de las obras se apoya en un conjunto de personas y de instituciones. No cuestiono, y por el contrario, me parece muy razonable, recurrir a una red de conocedores del tema como fuente de ampliación de la información tratándose en buena medida de la consagración de una pieza que por tan reciente puede ser desconocida en un radio amplio. La presencia de las instituciones abre el problema. La versión Europa se apoya en entidades vinculadas a la difusión de la cultura arquitectónica elegidas de una manera relativamente discrecional entre algunos países de ese continente. Para la versión América Latina se ha escogido en cambio a las instituciones de representación profesional de los países de la región. Es posible que esta leve asimetría se deba a la mayor dificultad para definir instituciones equiparables a las actuantes en la versión europea. Pero el problema derivado de esta diferencia es, para los resultados, más grave que en las anteriores. Porque la dinámica de las asociaciones profesionales, sus intereses, su relación con los asuntos culturales, no es en absoluto la misma que la que caracteriza a las instituciones de función preeminentemente cultural. Es natural que la elección de los trabajos más valiosos o significativos en el segundo caso se determine exclusivamente por criterios de calidad, como lo es que en el primero incidan consideraciones de equilibrio profesional y burocrático.

A partir de estas consideraciones es comprensible que esta segunda versión del premio (dejo de lado la primera por su condición especial) no resulte especialmente brillante. Nadie que conozca mínimamente el panorama de la arquitectura contemporánea en América Latina puede ignorar que Paulo Mendes da Rocha es uno de los tres o cuatro más grandes arquitectos del continente, y probablemente uno de los mejores del mundo. Nadie tampoco podría negar que tanto el primer premio como los trabajos que lo acompañan son piezas de interés. Pero me pregunto si es justo, conveniente y acertado "fomentar el conocimiento" de una figura excepcional como la de Paulo a través de su Pinacoteca, una obra sensible y bella pero que no sorprenderá a un espectador o lector de otras latitudes por su originalidad o por su especial condición. Y también tengo dudas de que por más meritoria que sea, la pequeña obra en Asunción del Paraguay pueda resistir una comparación internacional.
Daría la impresión de que el Jurado, la idoneidad de cuyos miembros no está aquí puesta en discusión, se vio obligado a elegir sometido a una red de múltiples condicionamientos, provenientes de las particularidades que he tratado de analizar brevemente. E hizo lo que mejor podía hacer: destacar un grupo de trabajos con criterios dispares: la trayectoria, el esfuerzo, la opera prima, la sensibilidad proyectual.

No quisiera ser mal entendido. No soy relativista cultural, y por ende no auspicio la construcción de procesos diferenciados por nación, por región o por comunidad étnica. Todo lo contrario, me parece que la elección de un esquema continentalista para los premios, precisamente porque es algo arbitraria y no basada en presuntas esencias, es perfectamente aceptable: un modo organizativo como cualquier otro. Pero si esto es así lo razonable es pensar que el correlato de un premio europeo es simplemente un premio americano. Y como se trata de juzgar en el marco de códigos de calidad que no están en la sangre sino en construcciones culturales compartidas y globales, sólo la capacidad de lograr una ponderación equilibrada -que incluye por supuesto el conocimiento profundo de lo que se juzga- debería regir el proceso de consagración. No sostengo que no existan o no deban existir aparentes asimetrías. Los esquemas organizativos no tienen por qué ser los mismos. Precisamente, porque no sólo es deseable que las versiones futuras logren ser más respetuosas de las similitudes que nos unen más de lo que creemos, sino porque también lo es que no dejen de hacer visibles las muchas diferencias que, nos gusten o no, nos separan. Ojalá que, en este sentido, el simétrico 2002 nos depare sorpresas. ARQ

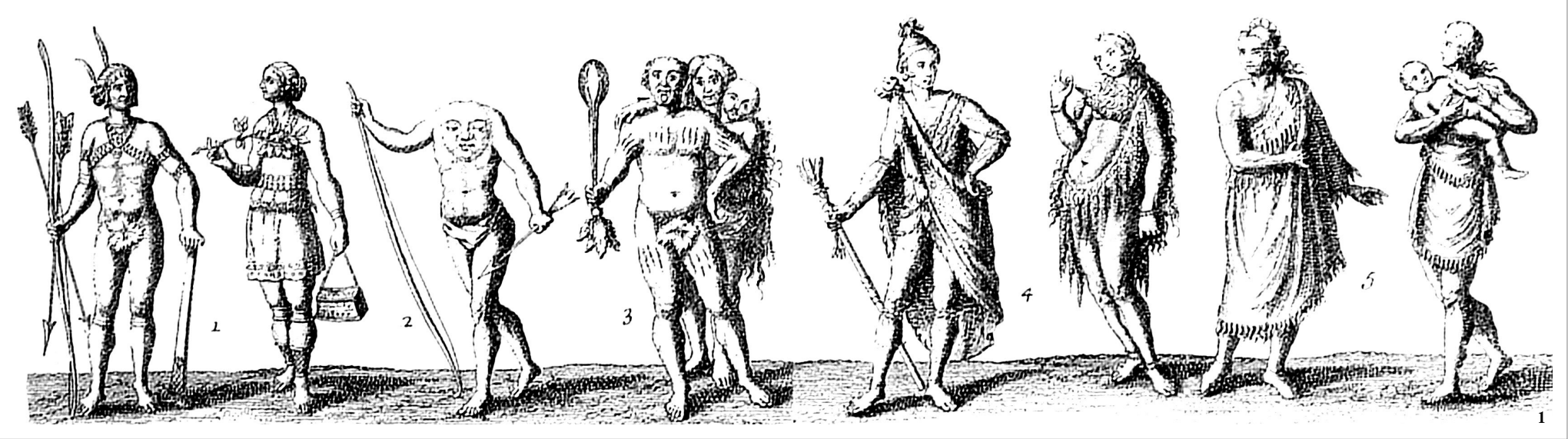

